\title{
CORPUS Corpus
}

Archivos virtuales de la alteridad americana

Vol 4, No 2 2014

Julio / Diciembre 2014

\section{Contribución a la medicina natural: Pohã Ñana, un Manuscrito inédito en Guaraní (Paraguay, S. XVIII)}

Contribution to Natural Medicine: Pohã Ñana. An Eighteenth Century Guarani

Language Manuscript

\section{Angélica Otazú Melgarejo}

\section{(2) OpenEdition}

\section{Journals}

Electronic version

URL: http://journals.openedition.org/corpusarchivos/1301

DOI: 10.4000/corpusarchivos.1301

ISSN: $1853-8037$

Publisher

Diego Escolar

Electronic reference

Angélica Otazú Melgarejo, «Contribución a la medicina natural: Pohã Nana, un Manuscrito inédito en Guaraní (Paraguay, S. XVIII) », Corpus [En línea], Vol 4, No 2 | 2014, Publicado el 22 diciembre 2014, consultado el 30 abril 2019. URL : http://journals.openedition.org/corpusarchivos/1301 ; DOI: 10.4000/corpusarchivos.1301

This text was automatically generated on 30 April 2019

Licencia Creative Commons: Atribución-NoComercial 2.5 Argentina (CC BY-NC 2.5 AR) 


\section{Contribución a la medicina natural: Pohã Nana, un Manuscrito inédito en Guaraní (Paraguay, S. XVIII)}

Contribution to Natural Medicine: Pohã Ñana. An Eighteenth Century Guarani Language Manuscript

Angélica Otazú Melgarejo

\section{EDITOR'S NOTE}

Fecha de recepción del original: 05/09/2014

Fecha de aceptación para publicación: 08/10/2014

\section{Introducción}

1 Presentamos un manuscrito del siglo XVIII, hasta hoy inédito, sobre medicina popular, escrito casi enteramente en guaraní, conservado en la Wellcome Library de Londres ${ }^{1}$, cuyo autor oficial es todavía desconocido. Pues por algún error en la interpretación de un epígrafe en guaraní se le atribuye al $\mathrm{H}^{\circ}$. Marcos Villodas.

Este manuscrito consta de una portada en grafía moderna añadida probablemente por los coleccionistas, que dice: POJHÃ ÑAÑA Materia Médica Misionera o Herbario de las Reducciones Guaraníes. Misiones Año de 1725 por MARCOS VILLODAS S.J3. (En adelante se lo denominará Ms. W. L. Londres). Existe además otra versión conservada en la Biblioteca Nacional de Madrid (BNM), que circulaba junto a un manuscrito de Gregorio López. El códice fue registrado en la BNM el $1^{\circ}$ de enero de 2004, con los siguientes datos: Autor personal: López, Gregorio (1542-1596). Libro de medicina en lengua guaraní; Libro de medicina por orden 
alphabetico, en español / Gregorio López ${ }^{4}$. (En adelante este texto se denominará Ms. B. N. Madrid). Ambos manuscritos estuvieron extraviados durante varios años.

En cambio, el manuscrito más conocido sobre medicina guaraní está escrito en castellano y pertenece al hermano Pedro de Montenegro (1663-1728); del mismo se conservan dos códices, en dos bibliotecas diferentes. En Buenos Aires, el que fue publicado por primera vez en forma completa en 1945 con el título de "Materia Médica Misionera", y reeditado por la Editorial Universitaria de la Universidad Nacional de Misiones, Posadas en 2007. El otro códice se conserva en la Biblioteca Nacional de Madrid cuyo título reza así: Libro primero y segundo de las propiedades y virtudes de los árboles y plantas de las Misiones y provincia del Tucumán, con algunas del Brasil y del Oriente. Año de 1710, publicado en 1995 por Carmen Martín Martín y José Luis Valverde, en Granada con el título de: La farmacia en la América Colonial: el arte de preparar medicamentos.

Debido al interés que ha despertado en los investigadores en los últimos años el estudio de la medicina guaraní de la época colonial, se intensificaron los análisis de los manuscritos referentes a la herboristería de las reducciones. Sin embargo, se centraron en los textos escritos en castellano, y solo pocos autores se refieren al Ms. W. L. Londres, atribuido a Marcos Villodas; tal es el caso de "La medicina misionera en Hispano-América y Filipinas durante la época colonial" de Carmen Sánchez Téllez, publicado en 1990 en España, y de Sabine Anagnostou, quien se refiere al mismo manuscrito en su trabajo publicado en 2005 en Alemania, titulado "Missionsmedizin und Missionspharmazie im kolonialen Amerika"5. Podemos decir, no obstante, que ambos estudios presentan más bien un conocimiento externo del manuscrito. Uno de los inconvenientes para analizar el Ms. W. L. Londres es el difícil acceso al guaraní clásico o el guaraní que se empleaba en las reducciones.

En este artículo daremos a conocer detalles más completos del Ms. W. L. Londres, cuya versión estamos estudiando desde hace cinco años. Las tres fases que hemos seguido son las siguientes: en vista a una comprensión panorámica del texto, se procedió a descifrar el significado global del MS y debido a la evolución de la lengua guaraní se realizó la transliteración en grafía actualizada del manuscrito completo; posteriormente se efectuó una interpretación y análisis de algunas características gramaticales del manuscrito, para iniciar la traducción; a continuación presentamos la descripción externa e interna del Ms. W. L. Londres, señalamos brevemente en qué contexto fue elaborado y sus posibles destinatarios, asimismo indagamos por qué el texto fue adjudicado a Villodas y exponemos dos ejemplos de terapias. Anexamos una tabla de contenido de la primera sección de ambos manuscritos con sus respectivas traducciones. Podemos decir que la autoría de los dos manuscritos sigue siendo una cuestión abierta y que los dos manuscritos sobre medicina en guaraní no serían los únicos. Agradecemos a todas las personas que de una u otra manera están colaborando para que culmine, exitosamente, el trabajo de la traducción ${ }^{6}$.

\section{Descripción externa e interna del Ms. W. L. Londres}

6 En primer lugar, describimos el aspecto externo del Ms. W. L. Londres ${ }^{7}$ Pohã Ñana. Está encuadernado en pergamino moderno, mide $19.5 \times 14.5 \mathrm{~cm}$. Comprende 60 folios, equivalente a 120 páginas, los títulos están enumerados en número arábigo, con una secuencia de f.1- f.205, aunque en esta secuencia se omiten varios números, mejor dicho, el manuscrito no contiene 205 recetas. Podemos agregar que en el índice no figuran todos 
los títulos de las recetas. Está escrito en lengua guaraní casi en su totalidad y es bastante legible. El texto se divide en tres secciones, a saber, la primera sección o primer libro contiene alrededor de treinta tratamientos de distintas dolencias, desde los dolores de cabeza hasta la viruela; la segunda sección o segundo libro contiene doce folios, sobre métodos terapéuticos de las mordeduras de víboras y picaduras de insectos, como también los tratamientos de ciertas afecciones de la piel, comezón, llagas y heridas por quemaduras, entre otros. La última sección del manuscrito consta de trece folios, sobre las instrucciones de la preparación y usos de diversas plantas medicinales y sus aplicaciones, y dos recomendaciones sobre la práctica de la sangría. Contiene una pequeña tabla que se titula: Nombres de algunas plantas en guaraní y castellano. Asimismo presenta una especie de apéndice con la inscripción \#, que consta de treinta títulos sobre tratamientos de dolencias y las actividades farmacológicas de las plantas.

7 En segundo lugar, puntualizamos la forma y fondo del Ms. W. L. Londres. Constatamos que este manuscrito posee unas normas de proceder para tratar las enfermedades, es decir, describe los síntomas de distintas dolencias; ofrece al usuario varios métodos terapéuticos e indica la duración de la administración de determinados medicamentos. Los tratamientos incluyen, generalmente, el cuidado especial del paciente en cuanto a la alimentación y reposo que debe guardar durante la convalecencia. Cabe mencionar que en la mayoría de los casos se recomienda la práctica de la sangría, la purga (laxante), la ventosa y ayuda (enema o lavativas), entre otras prácticas. Asimismo se tiene la posibilidad de substituir una planta medicinal por otra cuando la circunstancia así lo exige.

8 En cuanto a la lingüística, en la descripción de las recetas aparecen algunos usos de hispanismos. He aquí algunos ejemplos: las medidas como onza, libra y pesso real; en la nomenclatura de cifras se registran desde cuatro (aunque aparece también su equivalente en guaraní irundy), seis koterã ocho (seis u ocho, para señalar la cantidad de los ingredientes y la duración de la administración de ciertos remedios), mbohapy koterã cinco (tres o cinco) y el dúo once ndoze (once o doce) para designar una cantidad aproximativa; la denominación de los recipientes (vaso, frasco); en anatomía se registran las palabras hígado y corazón que en guaraní significan py'a. Probablemente prefirieron denominar estos órganos en castellano, puesto que py'a es una palabra polisémica y homógrafa, significa estómago, hígado, corazón y barriga; ingredientes como azúcar, conserva, aguardiente, azufre, entre otros.

9 Este texto es, además, una fuente muy importante para realizar investigaciones lingüísticas en el plano diacrónico. Puesto que se trata de un material con finalidad práctica, nos parece que la lengua guaraní utilizada se acerca mucho al uso coloquial de la época.

\section{3. ¿En qué contexto se elaboraron los manuscritos en guaraní?}

Los ámbitos abarcados por los misioneros jesuitas de los siglos XVII - XVIII, en la provincia del Paraguay, son la evangelización propiamente dicha, la urbanización, la herboristería y la curación como servicio al más próximo. Las obras sobre la medicina natural demuestran que los jesuitas estaban preparados en materia de historia natural y cirugía y que muchos de ellos habían practicado en hospitales europeos ${ }^{8}$. 
11 Es importante resaltar que los misioneros aplicaron un sistema terapéutico tradicional de la España del siglo XVI, como la sangría, purgantes, ventosa y la ayuda (enema), pero al mismo tiempo se beneficiaron de las distintas hierbas medicinales, según la clasificación conocida en Europa y las hierbas clasificadas y estudiadas en las selvas latinoamericanas; a falta de algunas plantas medicinales europeas, se sirvieron de las plantas locales, intercambiando los conocimientos botánicos con los autóctonos, para aliviar dolores de todo tipo y enfrentar grandes epidemias.

12 En este contexto actuaron muchos jesuitas que eran enfermeros empíricos, pero también otros instruidos en la materia como Pedro de Montenegro (1663-1728), Segismundo Asperger (1687-1772), el naturalista José Sánchez Labrador (1717-1798) y el cirujano hermano Marcos Villodas (1695-1741) con quien está relacionado el manuscrito Pohã Ñana. Conforme a los documentos históricos, este religioso era considerado un cirujano calificado, aunque no consta que haya estudiado medicina ${ }^{9}$, pero parece que no gozaba del mismo calificativo en cuanto a boticario "no sabe de botica y trueca los remedios y recetas, según dicen"10.

13 Marcos Villodas había nacido el $1^{\circ}$ de mayo de 1695 en Nanclares de Gamboa, Álava, País Vasco, era jesuita desde 1712. Llegó a Buenos Aires en $1717^{11}$. Se menciona que había pasado unos 20 años en las misiones ejerciendo su profesión; entre los años 1724 y 1735 ejerció en las misiones guaraníes del Uruguay ${ }^{12}$, luego fue destinado a la ciudad de Córdoba de Tucumán donde estuvo encargado de la botica hasta 1739. Falleció en Santa Fe en $1741^{\prime 13}$.

14 ¿Quiénes fueron los destinatarios del libro de recetas en guaraní? El libro Pohã Ñana o libro de los remedios, creemos que estaba dirigido especialmente a los enfermeros guaraníes y eventualmente a los futuros médicos indígenas, quienes, normalmente, aprendían a leer y a escribir en guaraní como se señala en el siguiente texto

No todos los niños eran instruidos en la lectura, escritura y en nociones de cálculo, sino tan sólo a aquéllos que pedía el bien de la ciudad. De entre ellos eran elegidos después el corregidor, los cabildantes, los magistrados, escribanos, procuradores públicos, los sacristanes y médicos ${ }^{14}$.

15 Teóricamente el texto podría haber estado dirigido también a los jesuitas enfermeros, encargados del cuidado de la salud en las reducciones ${ }^{15}$, que entraban en contacto con pacientes guaraní- hablantes y en esa circunstancia era menester un manual para tratar enfermedades en lengua indígena.

\section{4. ¿Quién o quiénes fueron los autores de los manuscritos: Ms. W. L. Londres y Ms. B. N. Madrid?}

16 La falta de claridad sobre la autoría de los manuscritos que estamos estudiando se remonta, probablemente, a la época de las reducciones, en la que los libros de remedios circulaban en manuscrito de pueblo en pueblo, en forma de cuadernos, sin especificar su autor, y estos cuadernos eran copiados o multicopiados para que las recetas no se perdieran. En este sentido se puede observar en el f. $35 \mathrm{v}$ del Ms. W. L. Londres, parte segunda del libro, donde se menciona solamente a la persona que usaba el material, y no propiamente a su autor:

17 "Quatia Ymomocoinda.Taš tetîrô ambuae pohÂno haguÂma rehegua Hacỉbaè P. H. Marcos Villodas rembiporutĭquè aracà̀" (f. 35v) (lit.: Segundo libro. Para curar a los enfermos de 
todos otros tipos de dolencias que utilizaba el $\mathrm{P}$. $\mathrm{H}^{\circ}$. Marcos Villodas). El título en castellano de la segunda parte de f.35v dice: "Parte Segunda / Del Libro de los Remedios del / $\mathrm{H} \stackrel{\circ}{\circ}$. Marcos Villodas".

Podemos observar que la traducción no corresponde al título en guaraní. Puesto que en guaraní se distingue la expresión rembiporutykue ${ }^{16}$ de rembiapokue ${ }^{17}$, rembiporuty indica cosa que se usa ordinariamente, utensilio, instrumento (kue indica ex-, pasado y araka'e es una partícula de tiempo pasado) y rembiapokue significa autoría, obra. El título en guaraní del manuscrito expresa claramente: libro que utilizaba el $\mathrm{H}^{\circ}$. Marcos Villodas (rembiporutykue araka'e). No hace mención a la autoría. Es más, el manuscrito no está firmado por ningún autor.

En el Ms. B. N. Madrid se repite el nombre del $\mathrm{H}^{\circ}$. Marcos, no obstante, no se menciona el apellido. "Quatia Porômboèha mirî Haçibaè rehegua MohÂ ypohano haguÂ Pe . Hermano Marcos rembiporutǐ" (p.1) (lit.: Pequeño libro de instrucciones o breve tratado sobre remedios para curar a los enfermos, del uso del Pe. Hermano Marcos). En este caso tampoco se refiere al autor sino al usuario: rembiporuty.

20 Aunque la autoría de esta versión nunca fue un tema de discusión, pues consta en la tercera hoja una dedicatoria autografiada con fecha 2 de junio de 1862, donde ya se le adjudicaba el texto a Gregorio López. Libro de Medicina, en los idiomas Guaraní y Castellano, Por el Venerable Gregorio Lopez, dedicado a mi joven amigo $D^{n}$. Antonio Tristán Ballester. Buenos Ayres, Junio 2 1862. Manuel E. Leyes (rúbrica). De todos modos, vemos que en la primera página de este manuscrito se hace referencia, a quien empleaba el texto ordinariamente, a un Pe. Hermano Marcos.

Asimismo, aparece en el segundo libro "Quatia imomocoînda poromongeta Taçĭ tetîrô ambuae pohÂno haguÂma rehe nicobaè Pay Hermano Marcos rembiporu" (p. 173), (lit.: Segundo libro prólogo sobre el tratamiento de otros tipos de remedios del uso del Padre Hermano Marcos). A diferencia de la segunda parte del Ms. W. L. Londres, este manuscrito no ofrece ninguna traducción al castellano. Pero tampoco está firmado por el tal Hermano Marcos. Por lo tanto, para ahondar nuestros conocimientos sobre las actividades en las misiones y por tratarse de documentos muy importantes, para el tema que estamos analizando, es preciso continuar la búsqueda de los verdaderos autores.

\section{Comparación interna de Ms. W. L. Londres y Ms. B. N. Madrid}

Nos preguntamos si ambos manuscritos provienen de una sola obra que circulaba en las reducciones. Una comparación interna entre los dos manuscritos nos permite comprobar que existe similitud de contenido, especialmente en los títulos de las recetas (ver la tabla del anexo).

Sin embargo, existe una pequeña diferencia en el léxico y la grafía. Véase en la siguiente tabla una pequeña muestra:

\begin{tabular}{|l|l|}
\hline Ms. W. L. Londres, f. 34 (f. 9v). ${ }^{18}$ & Ms. B. N. Madrid, p. 32. \\
\hline Açe ambuae cuèra taç̌̆ guasu amo & Açe amboaecuera taç̌̆ guasu amô \\
\hline
\end{tabular}




\begin{tabular}{|c|c|}
\hline oiporara ramo guguĭ omombuca otĩnguarupi, & oyporara ramo huguĭ omombuca otĩnguarupi, \\
\hline $\begin{array}{l}\text { coterã oyuru rupi hač haba mboagulye haguã } \\
\text { rehe }\end{array}$ & coterã oyuru rupi haçı̆ hague mboagulyebo; \\
\hline $\begin{array}{l}\text { ãnga ebocoibaè tuguř mombuca rehegua } \\
\text { ndaiquatiai chene: }\end{array}$ & $\begin{array}{l}\text { Anga ebocoibaè tugǔ mombuca rehegua } \\
\text { ndaiquatiay çene }\end{array}$ \\
\hline ambuae rehegua catu àçe rete mocãngǐbaè. & Amboae rehegua catu Açe rete mocãngǐbaè. \\
\hline Coterã oporoyucabaè taiquatia. & Coterã yyucabaè taiquatia. \\
\hline
\end{tabular}

A simple vista se puede distinguir la diferencia existente entre la grafía empleada en los dos textos, es decir, son evidentemente dos versiones diferentes, y probablemente de épocas diferentes. Un análisis exhaustivo puede arrojar más luces al respecto.

En cuanto a la originalidad del Ms. W. L. Londres como se ha señalado más arriba, en la secuencia de los folios (f. 1-f. 205) se omiten varios números y la comparación interna realizada con el Ms. B. N. Madrid, podrían indicar que estamos ante una copia de una tercera fuente en idioma guaraní.

\section{Breve descripción y análisis de dos terapias del manuscrito Pohã Ñana}

\subsection{Instrucciones para curar todo tipo de llagas viejas}

Exponemos la terapia de las llagas viejas, que figura en el manuscrito con el siguiente epígrafe: Porombo'eha ijai ymava'e tetĩrõ pohanõ haguã rehe teni kova'e (Ms. W. L. Londres f. 171, 39r.). Si analizamos la expresión ijai, veremos que está compuesta de ij "su" del castellano y la raíz ai. Esta raíz expresa en guaraní varias afecciones de la piel sin especificar su origen. Según los registros Montoya la palabra ai significa llaga y ai vyma es la llaga vieja:

"ai (-v-) llaga; che ai mi llaga »ij-; o-«; ai vyma llaga vieja; ai pyahu llaga nueva; ai péu llagas con materia; ai hovaso'i llaga que se va sanando, y criando cuero; che ai ai guitekóvo estoy llagado" (Montoya 2011, p. 17).

¿Cuáles son los síntomas y tratamientos de las llagas viejas? El Ms. W. L. Londres trata de esta enfermedad aparentemente nueva en la región. El autor describe detalladamente las características patológicas de las llagas viejas que pueden afectar a las personas, a continuación se transcribe la traducción del diagnóstico: "Hay varias formas de llagas viejas, las que afectan la parte interna del cuerpo, otras que producen hinchazón/ inflamación en la carne y otras que afectan la piel, otras producen pequeños agujeros en la carne del paciente, y producen pus". Del mismo modo el autor advierte que hay llagas viejas "que afectan los nervios y otras que afectan hasta los huesos". Y esto implica a su vez un cuidado especial: "En este caso el tratamiento tiene una pequeña diferencia, pero ambas clases requieren la purga".

El procedimiento de la terapia es un tema muy importante en el manuscrito, en este caso se presenta ordenadamente la terapia de la purga y la sangría: si se trata de llagas viejas 
debe repetirse varias veces la purga; si las llagas producen fiebre se debe practicar el sangrado, del lado del brazo, donde se padece la enfermedad, o ambos brazos uno o dos veces; si aumenta la hinchazón puede emplearse toda clase de remedios ya publicados.

El autor advierte sobre el comportamiento cambiante de las llagas: "Si se multiplican los tumores, entonces se tiene que prolongar el tratamiento para sanarse, debe aplicársele remedios más eficaces". No obstante el autor es consciente de que normalmente, "el tratamiento empleado sirve solo para mitigar el dolor".

Los procedimientos para eliminar los gusanos de la llaga son los siguientes: en primer lugar, se presenta la denominación del remedio, en este caso el tabaco, luego se explica su eficacia, se dan también otras opciones y por último se aconseja lavar con orina humana:

Si se le aplica el cocimiento del zumo de tabaco mata rápidamente todo tipo de gusanos de la carne. Y para que sea más eficaz cocer con tabaco o vinagre, y que se lave correctamente el gusano de la llaga con orín (humano), cuantas veces sea necesario.

A la par se recomienda emplear el zumo de juapekã como brebaje: "Hasy va'e o'yguahávamo, toiporu jepi juapekã rapo imbojypyre rykue. (lit.: Que el enfermo emplee como bebida el cocimiento del zumo de la raíz de juapekã).

La transpiración provocada con fines terapéuticos es una práctica muy antigua en la medicina tradicional. En la que por medio de un preparado de plantas medicinales se provocaba la sudoración del cuerpo del paciente. Esta práctica está registrada en el manuscrito analizado, sin embargo, se sirvieron de las plantas medicinales locales.

Como muestra, se transcribe la traducción "Que se le dé el siguiente remedio para que transpire su cuerpo, coger once o doce raíces de juapekã de una pulgada, diez raíces de tarope. Un puñado de kapi'i katĩ además un puñado de cardo santo". Se puede observar que el autor, además, de indicar las cuatro diferentes plantas, específica también la cantidad y la medida de cada uno de los ingredientes para preparar el medicamento que provocará la transpiración.

Luego señala el procedimiento de la preparación y cocción de los ingredientes, "frotar adecuadamente todas las raíces y machacarlas. Y cocer debidamente con cardo santo, se le añadirá seis vasos de agua. Después sacar del fuego". Por lo demás se indica, que el cocimiento se debe dejar reposar durante la noche y luego extraer el zumo, "y dejar reposar adecuadamente el cocimiento durante la noche, recalentarlo al amanecer. Y colar para extraer el zumo, luego colocarlo en un cántaro".

Por último, se facilita el modo y tiempo de administración de la medicina para conseguir el efecto deseado "esta medicina se debe emplear cuatro veces durante dos mañanas. Y por la tarde recalentarla debidamente, y después de cada toma del medicamento arropar adecuadamente al paciente a fin de provocar la transpiración".

\subsection{Hemorragia bucal y nasal}

37 Presentamos la terapia del flujo de la sangre, cuyo título en guaraní es el siguiente Porombo'eha ase tĩngua rupi, ha'e ijuru rupi guguy mombuka rasy pohanõ haguã rehegua (Ms. W. L. Londres f. 34, [9v-10r]) (lit.: Enseñanza sobre el tratamiento del flujo de la sangre de las narices y de la boca).

En este manuscrito se prescribe un tratamiento, por lo general, después de haber sido diagnosticado el tipo de la enfermedad. Presentamos a modo de ejemplo el siguiente 
texto: "Ase ambuae kuera tasy guasu amo oiporara ramo guguy omombuka otĩngua rupi, Koterã ojuru rupi hasy hava mboaguyje haguã rehe ãnga evokoiva'e tuguy mombuka rehegua ndaikuatiai chéne: ambuae rehegua katu ase rete mokangyva'e. Koterã oporojukava'e taikuatia". (Lit.: El tratamiento empleado cuando se padece alguna enfermedad grave, provocando el flujo de sangre de las narices, o de la boca para aliviar la dolencia, ese tipo de flujo de sangre no es necesario certificar, pero si se trata del que debilita a la persona o del que causa muerte, se debe certificar).

Curiosamente, para el tratamiento de la hemorragia también se recomendaba la sangría: "Y si requiere un tratamiento, hay que practicársele el sangrado varias veces en ambos brazos". Continúa la instrucción del procedimiento "después de esto restregar con un trapo hacia abajo, desde la cintura hasta los pies y sumergir las dos piernas en agua caliente para que la sangre circule hacia abajo".

¿Cuáles son los procedimientos para detener la hemorragia nasal? Por un lado se recomienda el uso del algodón quemado "si continua el flujo de sangre de las narices que se le introduzca algodón quemado en las fosas nasales". Por otro lado se recomienda la mezcla de arena con la sangre fresca: "Este es un remedio que detiene la hemorragia rápidamente, hacer mecha con un trapo y embarrar en la arena mezclada con la sangre recién derramada. Que esta mezcla se introduzca en las fosas nasales".

¿Cuál es el tratamiento de la hemorragia bucal? Ase juru rupi tuguy osẽ ramo opoti'a pegua tuguy ryru sorog hague rehe ijyvakutu rire Chejague rupi huguy mosẽvo toiporuuka ichupe Kova'e mohã, (literalmente: Si se padece hemorragia bucal debido a una rasgadura del vaso sanguíneo del pecho ${ }^{19}$ después de practicársele el sangrado, de acuerdo a lo indicado en mi obra, emplear el siguiente remedio).

En este caso el curandero tiene más de una opción para detener la hemorragia bucal derivada de una rasgadura del vaso sanguíneo del pecho y las hiervas recomendadas son rosa roja y llantén: "toipysy mokõi uru rupi'a morotĩngue ha'e toipyvu katu hese ombojese'a almidon kuiva'e peteĩ onz a mbyte ha'e Rosa pytã rykue. Koterã Ka'a juky rykue peteĩ vaso mirĩ toipyvu katu imoapomóvo. Kova'e mohã toiporu porara hasyva'e kucha pype here herévo nunga". (Tomar las claras de dos huevos y bien batidas mezclar con media onza de harina de almidón y el zumo de rosa roja. O revolverlas adecuadamente con un vasito de zumo de $k a$ 'a juky [llantén] de tal modo que el remedio se vuelva espeso). El autor indica además el modo de usar el medicamento "el enfermo debe ingerir continuamente este remedio con una cuchara, lamiéndolo poco a poco".

El texto ofrece además otro compuesto capaz de restañar el flujo de sangre

"Si se aplica una cucharada de la resina de ka'a vera con el zumo de ka'a juky cortará el flujo de sangre". Asimismo, indica que se debe "emplear aguara yvay del tamaño de un coquito disuelto. La corteza de ka'a vera emplear como brebaje o bebida, también ingerir el zumo de la corteza de sauce, o bien el zumo de la hoja". Es recomendable, según el autor, probar otros remedios cuando la corteza de ka'a vera o de sauce no detiene la hemorragia: "Si el paciente sigue padeciendo hemorragia, después de haber sido empledo dicho remedio que tome en ayunas un vasito de zumo de ka'a katu kangy. Este remedio normalmente es muy eficaz para detener el flujo de la sangre de la boca". Si el paciente presenta una mejoría después de ingerir el remedio indicado, se señala el modo y la duración del uso de la medicina: "si surte efecto ka'a katu kangy en pacientes que padecen hemorragia, que su cocimiento se emplee con la comida, e ingerir el zumo de este remedio durante seis u ocho días, por la mañana, como está indicado en mi escrito". 


\section{A modo de conclusión} algunas del Brasil y del Oriente. Año de 1710.

51 Montenegro, P. de (1945). Materia Médica Misionera. Nota preliminar de Raúl Quintana. Buenos Aires: Imprenta de la Biblioteca Nacional.

ANEXO. Transcripción de la tabla de contenido de la primera sección de ambos manuscritos con sus respectivas traducciones realizada por la autora.

\begin{tabular}{|c|c|c|c|}
\hline Tabla: Ms. W. L. Londres & Traducción literal & $\begin{array}{l}\text { Tabla: Ms. B. N. } \\
\text { Madrid }\end{array}$ & Traducción literal \\
\hline $\begin{array}{l}\text { Poromongeta mirî haçǐbaè } \\
\text { rehe ñangarecoha upe } \\
\text { guarÂma f. } 1 .\end{array}$ & $\begin{array}{l}\text { Breve discurso sobre } \\
\text { el cuidado de los } \\
\text { enfermos. }\end{array}$ & $\mid \begin{array}{lr}\text { Quatia } & \text { Porôboèha } \\
\text { mirî } & \text { haçǐbaè } \\
\text { rehegua } & \text { Moĥ̂A } \\
\text { tetîrô } & \text { rehegua } \\
\text { Ymombeù } & \text { haba } \\
\text { tenicobaè } & \end{array}$ & $\begin{array}{l}\text { Breve tratado sobre } \\
\text { los } \\
\text { Presentación enfermos. } \\
\text { diferentes tipos de } \\
\text { remedios. }\end{array}$ \\
\hline
\end{tabular}




\begin{tabular}{|c|c|c|c|}
\hline $\begin{array}{l}\text { 1. Poromboèha acÂraçî } \\
\text { tetîrô reheguaete f. } 2 \text {. }\end{array}$ & $\begin{array}{l}\text { Instrucción sobre } \\
\text { varios tipo de dolor } \\
\text { de cabeza }\end{array}$ & $\begin{array}{l}1 . \quad \text { Poromboèha } \\
\text { acÂraçǐ r tetîrô } \\
\text { rehegua pag. } 2 .\end{array}$ & $\begin{array}{l}\text { Instrucción sobre } \\
\text { varios tipo de dolor de } \\
\text { cabeza. }\end{array}$ \\
\hline $\begin{array}{l}\text { 2. AcÂraçı̌ ambuae } \\
\text { pohâno haguÂ f. } 5 .\end{array}$ & $\begin{array}{l}\text { Para curar otros } \\
\text { tipos de dolores de } \\
\text { cabeza. }\end{array}$ & $\begin{array}{ll}\text { 2. Conico } & \text { acÂraçǐ } \\
\text { ambuae } & \text { pohÂno } \\
\text { haguÂ. } 4 . & \end{array}$ & $\begin{array}{l}\text { He aquí el tratamiento } \\
\text { para otros tipos de } \\
\text { dolores de cabeza. }\end{array}$ \\
\hline $\begin{array}{l}\text { 3. AcÂraçǐ mbaè ambuae } \\
\text { rehegua pohÂnoha f. } 6\end{array}$ & $\begin{array}{lr}\text { Para tratar } & \text { el dolor } \\
\text { de cabeza } & \text { causado } \\
\text { por } & \text { dolor } \\
\text { estomacal... } & \end{array}$ & & \\
\hline $\begin{array}{l}\text { 4. Mohâ reta acÂraçĭ } \\
\text { tetîrô pohanôha f. } 10 .\end{array}$ & $\begin{array}{lr}\text { Los remedios } & \text { para } \\
\text { curar diversos } & \text { tipo } \\
\text { de dolores } & \text { de } \\
\text { cabeza. } & \end{array}$ & $\begin{array}{l}\text { 3. Conico mohâ acÂ } \\
\text { raçı̆ tetîrô pohÂ } \\
\text { noha. } 6 .\end{array}$ & $\begin{array}{l}\text { He aquí los remedios } \\
\text { que curan diversos } \\
\text { tipos de dolores de } \\
\text { cabeza. }\end{array}$ \\
\hline $\begin{array}{l}\text { 5. Omano çapřabae raÂnga } \\
\text { pohanôha f.11. }\end{array}$ & $\begin{array}{l}\text { Para el tratamiento } \\
\text { del desvanecimiento } \\
\text { o mal del corazón. }\end{array}$ & $\begin{array}{lr}4 . & \text { Poromboèha } \\
\text { omanô } & \text { çapřàbaè } \\
\text { râÂnga } & \text { pohanôha } \\
\text { nicobaè } 7 .\end{array}$ & $\begin{array}{l}\text { Instrucción para el } \\
\text { tratamiento } \\
\text { desvanecimiento o mal } \\
\text { del corazón. }\end{array}$ \\
\hline $\begin{array}{l}\text { 6. Poromboèha Yepep̌̃ } \\
\text { raçĭ rehegua, CoterÂ ace } \\
\text { rayu mobĭrÂquÂ f. } 15 .\end{array}$ & $\begin{array}{l}\text { Instrucción sobre el } \\
\text { tratamiento de las } \\
\text { venas dislaceradas / } \\
\text { inflamación de las } \\
\text { venas. }\end{array}$ & $\begin{array}{l}\text { 5. Poromboèha } \\
\text { Yepepĭ raçĭ rehegua, } \\
\text { coterÂ açe rayu } \\
\text { mobĩrÂqua pohanô } \\
\text { ha ni cobaè } 11 .\end{array}$ & $\begin{array}{l}\text { Instrucción sobre el } \\
\text { tratamiento de las } \\
\text { venas dislaceradas/ } \\
\text { inflamación de vena. }\end{array}$ \\
\hline $\begin{array}{l}\text { 7. Teça raçĭ tetîrô pohanô } \\
\text { haguÂ rehegua f. } 22 \text {. }\end{array}$ & $\begin{array}{l}\text { La curación de todo } \\
\text { tipo de mal de ojos. }\end{array}$ & $\begin{array}{l}\text { 6. Poromboèha Teça } \\
\text { raçî tetîrô pohanô } \\
\text { haguÂ } \\
\text { rehegua rehegua } \\
\text { nicobaè } 18 .\end{array}$ & $\begin{array}{l}\text { Instrucción para curar } \\
\text { de todo tipo de mal de } \\
\text { ojos }\end{array}$ \\
\hline $\begin{array}{l}\text { 8. Apřçaraçŭ, ap̌çarebobo, } \\
\text { haè ap̌çaey̆baè pohanô } \\
\text { haguÂ.f. } 28 .\end{array}$ & $\begin{array}{l}\text { Para curar dolores } \\
\text { de oídos, hendidura } \\
\text { de oídos y la sordera. }\end{array}$ & $\begin{array}{l}\text { 7. Poromboèha Ap } \\
\text { Içça ra çî haè Apǐça } \\
\text { rebobog, haè apǐç } \\
\text { aeỹ } \\
\text { baè pohanô } \\
\text { rehegua. 26. }\end{array}$ & $\begin{array}{l}\text { Instrucción para curar } \\
\text { dolores de oídos, } \\
\text { hendidura de oídos y } \\
\text { la sordera. }\end{array}$ \\
\hline $\begin{array}{l}\text { 9. Açe tĩngua rupi, haè } \\
\text { yyuru rupi tugur } \\
\text { mombucarač } \text { pohano } \\
\text { hagua rehegua f. } 34 .\end{array}$ & $\begin{array}{l}\text { Para curar } \\
\text { hemorragia } \\
\text { bucal. }\end{array}$ & $\begin{array}{l}\text { 8. Poromboèha açe } \\
\text { tîngua } r \text { rupi } \\
\text { Yjururupi } \quad \text { guguř } \\
\text { mombuca pohÂno } \\
\text { haguÂma rehe } 32\end{array}$ & $\begin{array}{l}\text { Instrucción para curar } \\
\text { hemorragia nasal y } \\
\text { bucal. }\end{array}$ \\
\hline $\begin{array}{l}\text { 10. AcerÂŷ raç̌ poh Âno } \\
\text { hagua f. } 36\end{array}$ & $\begin{array}{l}\text { Para curar dolores } \\
\text { de muelas. }\end{array}$ & $\begin{array}{l}\text { 9. Poromboèha açe } \\
\text { raî raçǔ poh Âno } \\
\text { haguÂma } 34\end{array}$ & $\begin{array}{l}\text { Instrucción para curar } \\
\text { dolores de muelas. }\end{array}$ \\
\hline
\end{tabular}




\begin{tabular}{|c|c|c|c|}
\hline $\begin{array}{l}\text { 11. Açe yač̌o rač̆, ace } \\
\text { yuruay abe pohanô haguÂ } \\
\text { rehegua f. } 39 \text {. }\end{array}$ & $\begin{array}{l}\text { Para curar dolor de } \\
\text { garganta y de igual } \\
\text { forma la llaga bucal. }\end{array}$ & 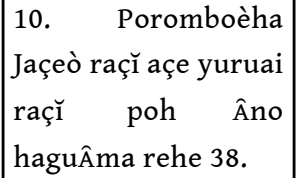 & $\begin{array}{l}\text { Instrucción para curar } \\
\text { dolor de garganta y la } \\
\text { llaga bucal. }\end{array}$ \\
\hline $\begin{array}{l}\text { 12. Uù bač̌ pohÂnô haguÂ } \\
\text { f. } 47 \text {. }\end{array}$ & $\begin{array}{l}\text { Para curar la tos, el } \\
\text { catarro. }\end{array}$ & $\begin{array}{l}\text { 11. Poromboèha } \\
\text { Uùbaç̌̃ pohano hagu } \\
\text { Â } 47 .\end{array}$ & $\begin{array}{l}\text { Instrucción para curar } \\
\text { la tos, el catarro. }\end{array}$ \\
\hline $\begin{array}{l}\text { 13. Ace pĭtiacutu, hae } \\
\text { pŭtiacutu mbĭte raça } \\
\text { pohanô hagua rehegua } \mathrm{f} \text {. } \\
52 .\end{array}$ & $\begin{array}{l}\text { (Sobre el tratamiento } \\
\text { del dolor agudo del } \\
\text { pecho y el dolor agudo } \\
\text { que atraviesa el centro } \\
\text { pecho. / Enfermedades } \\
\text { pectorales?) }\end{array}$ & $\begin{array}{lr}12 . & \text { Poromboèha } \\
\text { Pytià } & \text { cutumbù } \\
\text { teraça } & \text { pohÂno hagu } \\
\text { Âma rehe } 52 .\end{array}$ & $\begin{array}{l}\text { (Instrucción sobre el } \\
\text { tratamiento del dolor } \\
\text { agudo que atraviesa el } \\
\text { centro del pecho./ } \\
\text { Enfermedades } \\
\text { pectorales?) }\end{array}$ \\
\hline $\begin{array}{l}\text { 14. Mbràrač̌ pohanô } \\
\text { haguÂ rehegua f. } 57 .\end{array}$ & $\begin{array}{l}\text { Sobre el tratamiento } \\
\text { de dolor de } \\
\text { estómago hígado. }\end{array}$ & $\begin{array}{l}\text { 13. Poromboèha } \mathrm{Mb} \\
\text { là raçî pohÂno hagu } \\
\text { Âma } 58 .\end{array}$ & $\begin{array}{l}\text { Instrucción sobre el } \\
\text { tratamiento del dolor } \\
\text { de estómago/hígado. }\end{array}$ \\
\hline $\begin{array}{l}\text { 15. açe guê̂ mbacî } \\
\text { pohano hagua rehe(gua) f. } \\
58 .\end{array}$ & $\begin{array}{l}\text { Sobre el tratamiento } \\
\text { de vómito. }\end{array}$ & $\begin{array}{l}\text { 14. Poromboèha açe } \\
\text { guê̂ mbaç̌̆ poh Âno } \\
\text { haguarehe } 66 .\end{array}$ & $\begin{array}{ll}\text { Instrucción sobre el } \\
\text { tratamiento } & \text { de } \\
\text { vómito. } & \end{array}$ \\
\hline $\begin{array}{l}\text { (16) /18/ Poromboè ha á } \\
\text { çe r re rendapegua rač̀ } \\
\text { pohanô } \\
\text { rehegua. } 59 .\end{array}$ & $\begin{array}{l}\text { Instrucción sobre el } \\
\text { tratamiento de } \\
\text { dolores de vientre. }\end{array}$ & $\begin{array}{l}\text { 15. Poromboèha açe } \\
\text { rǔe rÊndapegua haçǐ } \\
\text { pohano ha guÂma } \\
\text { rehe } 71 .\end{array}$ & $\begin{array}{l}\text { Instrucción sobre el } \\
\text { tratamiento de dolores } \\
\text { de vientre. }\end{array}$ \\
\hline $\begin{array}{l}(17) / 19 / \text { Poromboèha } \\
\text { tǐetetîrô pohanô haguÂ } \\
\text { rehegua. } 60\end{array}$ & $\begin{array}{l}\text { Intrucción sobre el } \\
\text { tratamiento de la } \\
\text { diarrea / disentería. }\end{array}$ & $\begin{array}{l}\text { 16. Poromboèha Tĭe } \\
\text { tetîro pohÂno } \\
\text { haguÂma } 76 .\end{array}$ & $\begin{array}{l}\text { Intrucción sobre el } \\
\text { tratamiento de la } \\
\text { diarrea / disentería. }\end{array}$ \\
\hline $\begin{array}{l}\text { (18) /23/ Poromboèha } \\
\text { taçoporoçuù açepĭapegua, } \\
\text { haè yñŭî̃ mengua } \\
\text { pohanôha tenico bae. } 70 .\end{array}$ & 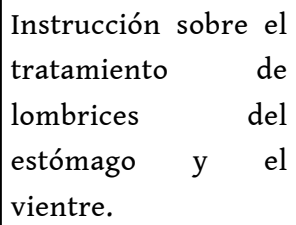 & $\begin{array}{l}17 . \quad \text { Poromboèha } \\
\text { taço çuù raçî haè } \\
\text { pĭàpegua, haè yñủî̌ } \\
\text { mÊngua pohÂnoha } \\
\text { nicobaè. } 93 .\end{array}$ & \begin{tabular}{|lr} 
Instrucción & sobre \\
tratamiento & de \\
lombrices & del \\
estómago y el vientre.
\end{tabular} \\
\hline $\begin{array}{l}(19) / 24 / \text { Poromboèha } \\
\text { açetî raçî rehegua haè } \\
\text { yquaru eỹ remo pohanô } \\
\text { haguÂma teni cobaè. [sin } \\
\text { número] }\end{array}$ & $\begin{array}{l}\text { Instrucción } \\
\text { tratamiento de }\end{array}$ & $\begin{array}{lrr}18 . & \text { Poromboèha } \\
\text { açetĩ } & \text { raçî } & \text { rehegua, } \\
\text { haè } & \text { yquarueỹ } & \text { rehe } \\
\text { pohÂno } & \text { haguÂA } \\
\text { nicobaè. } 99 .\end{array}$ & $\begin{array}{l}\text { Instrucción sobre el } \\
\text { tratamiento de la } \\
\text { infección de la vía } \\
\text { urinaria y retención } \\
\text { de orina. }\end{array}$ \\
\hline $\begin{array}{l}(20) / 26 / \text { Poromboèha } \\
\text { cuña raç̌ porara rehegua } \\
\text { teni cobaè. } 72\end{array}$ & $\begin{array}{lll}\text { He aquí } & \text { la } \\
\text { instrucción sobre la } \\
\text { menstruación de } & \text { la } \\
\text { mujer. } & \end{array}$ & $\begin{array}{l}\text { 19. Poromboèha } \\
\text { cuña raçî porara } \\
\text { rehegua } 110 .\end{array}$ & $\begin{array}{l}\text { Instrucción sobre la } \\
\text { menstruación de la } \\
\text { mujer. }\end{array}$ \\
\hline
\end{tabular}




\begin{tabular}{|c|c|c|c|}
\hline $\begin{array}{l}(21) \text { /27/ Poromboèha } \\
\text { MitÂ à haguÂ pritîbô } \\
\text { rehegua teni cobaè. } 73\end{array}$ & $\begin{array}{lll}\text { Instrucción } & \text { sobre la } \\
\text { asistencia } & \text { en } & \text { el } \\
\text { parto. } & & \end{array}$ & $\begin{array}{l}20 . \quad \text { Poromboèha } \\
\text { MitÂ àhag }{ }^{\widehat{A}} \text { pŭtĭbô } \\
\text { rehegua } 113 .\end{array}$ & $\begin{array}{l}\text { Instrucción sobre la } \\
\text { asistencia en el parto. }\end{array}$ \\
\hline $\begin{array}{l}(22) \text { /27/ MohÂ MitÂg } \\
\text { omanôbaècuè oçĭrǐepe } \\
\text { mbogueyĭ haguÂrehe, haè } \\
\text { hupague mbogueyǐbo } \\
\text { râno. 74. }\end{array}$ & 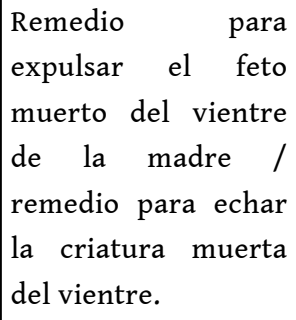 & $\begin{array}{l}\text { 21. MohÂ Mit Âng } \\
\text { omanobaècue } \\
\text { oçı̌rı̌epe mboguey } \\
\text { haguÂ rehe, haè } \\
\text { hupague } \\
\text { mbogueyı̌bo rÂno } \\
117 .\end{array}$ & $\begin{array}{l}\text { Remedio para expulsar } \\
\text { el feto muerto del } \\
\text { vientre de la madre. }\end{array}$ \\
\hline $\begin{array}{l}(23) / 28 / \text { Poromboèha } \\
\text { MitÂç̌̆, omembĭ mboa rire } \\
\text { haçî poraratî rehegua, haè } \\
\text { huguĭ mombuca raçî } \\
\text { pohanô haguÂ rehe ranô. }\end{array}$ & $\begin{array}{l}\text { Instrucciones sobre } \\
\text { el tratamiento de la } \\
\text { etapa del puerperio } \\
\text { y el tratamiento de } \\
\text { la hemorragia. }\end{array}$ & 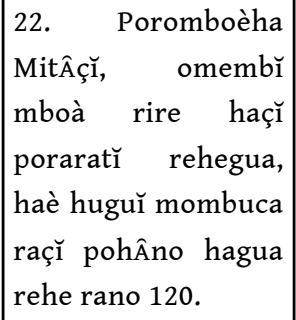 & $\begin{array}{l}\text { Instrucciones sobre el } \\
\text { tratamiento de la } \\
\text { etapa del puerperio y } \\
\text { el tratamiento de la } \\
\text { hemorragia. }\end{array}$ \\
\hline $\begin{array}{l}\text { (24) } 16 . \text { MitÂ oyac ǐeỹme } \\
\text { ya potaramo ymombĭta } \\
\text { haguÂ rehegua f. } 121 .\end{array}$ & $\begin{array}{l}\text { Sobre el tratamiento } \\
\text { para evitar el } \\
\text { nacimiento } \\
\text { prematuro. }\end{array}$ & $\begin{array}{l}23 . \quad \text { Poromboèha } \\
\text { MitÂ oyaç̌eỹme oà } \\
\text { potarÂmo } \\
\text { ypohÂnoha ymomb } \\
\text { ĭta haguÂ rehe } \\
\text { nicobaè. } 133 .\end{array}$ & $\begin{array}{l}\text { Instrucciones sobre el } \\
\text { tratamiento para } \\
\text { evitar el nacimiento } \\
\text { prematuro. }\end{array}$ \\
\hline $\begin{array}{l}\text { (25) 17. Tacubač reco } \\
\text { quaa haguÂ rehegua f. } 128\end{array}$ & $\begin{array}{l}\text { Sobre el tratamiento } \\
\text { de la calentura. }\end{array}$ & $\begin{array}{l}24 . \quad \text { Poromboèha } \\
\text { Tacu baçî reco quaa } \\
\text { haguÂA rehegu Âma } \\
\text { rehe } 136\end{array}$ & $\begin{array}{l}\text { Instrucciones sobre el } \\
\text { tratamiento de la } \\
\text { calentura. }\end{array}$ \\
\hline $\begin{array}{l}\text { (26) 18. Tacubacǐ pohano } \\
\text { f. } 129 .\end{array}$ & $\begin{array}{l}\text { Para curar la } \\
\text { calentura/ fiebre } \\
\text { ardiente. }\end{array}$ & $\begin{array}{lc}25 . & \text { Poromboèha } \\
\text { Tacubaçî } & \text { pohano } \\
\text { rehegua } & \text { nicobaè } \\
142 . & \end{array}$ & $\begin{array}{lc}\text { Instrucción } & \text { sobre el } \\
\text { tratamiento } & \text { de la } \\
\text { calentura/ } & \text { fiebre } \\
\text { ardiente. } & \end{array}$ \\
\hline $\begin{array}{l}\text { (27) } 19 . \text { Mbiruà racĭ } \\
\text { pohanô haguÂ rehegua f. } \\
\text { 139. }\end{array}$ & $\begin{array}{l}\text { Sobre el tratamiento } \\
\text { de la viruela }\end{array}$ & $\begin{array}{l}/ 161 / 26 . \\
\text { rač pohano haba. } \\
\text { corami }\end{array}$ & $\begin{array}{l}\text { Sobre el tratamiento } \\
\text { de la viruela. }\end{array}$ \\
\hline
\end{tabular}




\section{BIBLIOGRAPHY}

Anagnostou, S. (2005). Missionsmedizin und Missionspharmazie im kolonialen Amerika. Wiesbaden: Harrassowitz. In Meier, Johannes (Hg.) 2005 Sendung - Eroberung - Begegnung. Franz Xaver, die Gesellschaft Jesu und diekatholische Weltkirche im Zeitalter des Barock, Wiesbaden: Harassowitz-Verlag, 446 S. pp. 261-292.

Canton, E. (1928). Historia de la Medicina del Río de la Plata. Tomo I, Madrid.

Furlong, G. (1947). Naturalistas argentinos durante la dominación hispánica. Buenos Aires: Huarpes.

Martín Martín, C. y Valverde, J. L. (1995). La farmacia en la América colonial: el arte de preparar medicamentos. Granada: Universidad de Granada.

Montoya, A. Ruiz de (2011). Tesoro de la lengua guaraní. Madrid 1639. Edición Facsimilar, Leipzig, Oficina y funderia de W. Drugulin1876. Asunción: CEPAG.

Melià, B. Antología de textos coloniales, en prensa.

Otazú, A. (2006). Práctica y semántica en la evangelización de los guaranies del Paraguay (S. XVI-XVIII). Asunción: CEPAG.

Peramàs, J. M. (2004). Platón y los guaraníes. Nueva versión del original latino por Francisco Fernández Pertíñez y Bartomeu Melià. Asunción: CEPAG.

Sánchez Téllez, C. (1990). La medicina misionera en Hispano-América y Filipinas durante la época colonial. Estudios de historia social y económica de América, 6, 33-40.

\section{NOTES}

1. Fue adquirido en 1962 junto con otros documentos de la colección del mejicano doctor Francisco Guerra, médico y uno de los bibliófilos más representativos de nuestro tiempo e historiador de la medicina de América Latina.

2. En la portada figura POJHÃ ÑANA. Pero ñaña hace referencia a maldad, diablo, y lo correcto es ñana que significa hierba, yuyo.

3. Estamos preparando en el marco del Proyecto Kuatia Yma guare (PeKY) de la Universidad de Kiel una edición bilingüe (guaraní y castellano) para ponerlo finalmente al servicio de la comunidad científica.

4. Fue localizado en marzo de 2013 por Capucine Boidin, coordinadora del Proyecto LANGAS Langues Générales d'Amérique du Sud (Quechua, Guarani) XVI et XIX ${ }^{\mathrm{e}}$ siècles, Paris.

5. Anagnostou, S. 2005, pp. 261-292.

6. Al antiguo equipo de Kiel, Martín Ramírez Machuca-pe che pytyvõ haguére ahesa'ỹijo haguã pe kuatiahai ha Karai Harald Thun-pe katu ome'ẽ haguére chéve ko kuatia ha ore mongyre'ỹ haguére roikuaa haguã mba'épa he'i ko kuatiápe. A Federico Otazú Melgarejo por su colaboración en la transliteración. A la colega Capucine Boidin y al equipo de LANGAS por facilitar un manuscrito más sobre medicina guaraní.

7. Al final del manuscrito, figura esta nota "este «Codice Villodas» es el unico manuscrito medico guarani conocido y se refiere a las propiedades terapéuticas de las plantas de las misiones 
jesuiticas en el Paraguay. El padre Furlong S.J. no llegó a verle. Procede de la colección Guarani de Moisés S. Bertoni, Asunción, Paraguay y fue adquirido del prf. Guillermo Tell Bertoni en Asunción, el 5 de mayo 1957".

8. Véase Canton 1928, p. 275.

9. Véase Sánchez Téllez 1990, p. 36.

10. Furlong 1947, p. 97.

11. Melià, B. Antología, texto aún inédito.

12. Véase Furlong 1947. pp. 62-63, 80-81, 96-97.

13. Melià, B. Antología, texto aún inédito.

14. Peramàs 2004, p. 77.

15. Al inicio de la fundación de las misiones, los hermanos legos y algunos padres ejercían como boticarios y cirujanos a la vez (Véase Canton 1928. p. 275).

16. Sustantivo triforme: Tembiporu, rembiporu, hembiporu. Utensilio, instrumento. Ne rembiporu: tu utensilio; hembiporu: su utensilio.

17. Sustantivo triforme:Tembiapo, rembiapo, hembiapo. Tembiapokue, ej.: Este libro es de mi autoría: ko kuatia che rembiapokue; este libro es de su autoría: ko kuatia hembiapokue.

18. Lit.: El tratamiento empleado cuando se padece alguna enfermedad grave, provocando el flujo de sangre de las narices, o de la boca para aliviar la dolencia, ese tipo de flujo de sangre no es necesario certificar, pero si se trata del que debilita a la persona o del que causa muerte, se debe certificar (Ms. W. L. Londres, f. 34 [f. 9v]).

19. Lit.: opoti'a pegua tuguy ryru: vaso de sangre ubicado en el pecho.

20. Véase Otazú 2006, pp.93-192.

\section{ABSTRACTS}

After five years of transliterating, translating and research, we have been encouraged to write a brief description of the Poha Ñana manuscript (1725) currently attributed to Marcos Villodas. We will also present in brief another manuscript (no date) called Libro de medicina en lengua guaraní. We will then mention some of the similarities and differences between the two codices. Later we will show the translation and analysis of two examples of therapies for specific diseases that can be found in Ñana Pohã. It is unclear at this time whether the two extant sources are in fact drawn from a third manuscript which may have circulated among the villages of the Jesuit Reductions, within the original territory of Paraguay.

Después de cinco años de trabajo de transliteración, traducción e investigación hemos decidido hacer una breve presentación para describir en forma externa e interna el manuscrito Pohã Ñana (1725) atribuido a Marcos Villodas y presentar someramente también otro manuscrito denominado el Libro de medicina en lengua guaraní, sin fecha. Señalamos brevemente la similitud y la diferencia de contenido de los dos códices. Exponemos la traducción y análisis de dos ejemplos de terapias para enfermedades específicas en Pohã Ñana. No está claro en la actualidad si las fuentes existentes son extraídas de un tercer manuscrito, que pudo haber circulado en los pueblos de las reducciones jesuíticas del antiguo territorio paraguayo. 
INDEX

Keywords: History, South American medicine, codices in Guarani, Paraguay

Palabras claves: Historia, medicina sudamericana, códice en guaraní

\section{AUTHOR}

ANGÉLICA OTAZÚ MELGAREJO

Universidade Federal da Grande Dourados, MS-Dourados - MS, Brasil

Correo electrónico: potykuru@hotmail.com 